\title{
Environmental and health issues
}

\author{
Junshi Miyamoto \\ President, Division of Chemistry and the Environment, IUPAC*
}

\begin{abstract}
The environmental behavior of a chemical is governed by its intrinsic physicochemical properties as well as by characteristic features of the environmental compartments, including their metabolic activities. This inextricable network of interactions contribute to degradation and mineralization of organic compounds. Under the circumstances that the chemical is lipophilic and/or resistant against chemical and biological degradation, it tends to be transported to other environmental compartments and/or subject to bioaccumulation. The adverse effects of chemicals on humans and organisms in the environment are assessed by a variety of toxicological studies to establish ADI (acceptable daily intake in man) or PNEC (predicted no observed effect level in organisms), in comparison with the calculated exposure levels, based on the environmental behavior of chemicals.
\end{abstract}

\section{EMISSION OF CHEMICALS IN THE ENVIRONMENT}

Emission of chemical substances in the environment due to natural and anthropogenic activities constitutes the primary process of their environmental contamination. It is dependent on various factors: For industrial chemicals production volume - e.g. from thousand tonnes/year of pesticides to several ten thousand tonnes of styrene or butadiene monomer - life stage (life cycle), i.e. production, formulation, processing (for professional use, private use, etc.), and application which may result in use in closed systems as intermediate of production process, non-dispersive use, or wide dispersive use in case of pesticides which are $100 \%$ applied intentionally to the environment. A certain portion of these chemicals and their derivatives may be emitted during waste disposal processes as well. Not a negligible number of biogenic substances like methyl chloride ( 5 million tonnes/year by marine algae, giant kelp, wood-rotting fungi, etc.), chloroform (by marine algae, white cedar, lemon, etc.) and bromodichloromethane (by marine algae) just as simple examples, are released also from a large number of natural sources [1]. In addition, nature is an excellent producer of complex molecules, including carcinogenic aflatoxins and sterigmatocystin as well as a variety of toxic constituents in plants even like carcinogenic pyrrolizidine alkaloids. Although these naturally occurring substances are not widely emitted into the environment, they often represent significant examples of biologically active (hazardous) substances, since humans and animals are exposed to them under certain circumstances.

\section{BEHAVIOR IN THE ENVIRONMENT [2-7]}

Chemical substances emitted in the environment are distributed/translocated in its compartments and/or transformed due to inextricable network of interaction of their physicochemical properties with a variety of environmental parameters as shown in Table 1. (N.B. Emission itself is to some degrees dependent on physicochemical properties of chemicals.)

Thus, first of all, the knowledge on physicochemical properties of chemicals is essential for understanding and predicting the mobility, degradation (abiotic and biotic), as well as the accumulation potential (abiotic and biotic) in the environment.

\subsection{Mobility}

The mobility of a substance in the environment defined as the ability of a substance to move within the environment, is determined by the distribution behavior of a substance between the environmental media

*IUPAC Secretariat, 2-3 Pound Way, Templars Square, Cowley, Oxford OX4 3YF, UK. 
Table 1 Factors associated with environmental behavior of chemical substances

Physicochemical properties of chemical substances

structure, molecular weight, melting point, absorption spectra in UV and visible light regions, boiling point, vapor pressure, water solubility, $P_{\text {ow }}$ (octanol/water partition coefficient), hydrolyzability.

\section{Environmental parameters}

General:

geography and climate/meteorology (latitude, altitude, sunlight intensity, rainfall, wind, temperature, humidity, etc.) air:

chemical composition (presence of contaminants), suspended particles, aerosols water:

$\mathrm{pH}$, suspended materials, soluble inorgamics and orgamics, biota (fish, crustaceans, snails, insect larvae,

planktons, benthos, etc.)

soil and sediment:

clay mineral, cation exchange capacity $(\mathrm{Al}, \mathrm{Mg}, \mathrm{Cu}$, etc.)

organic matter content

$\mathrm{pH}$

redox potential

biota (terrestrial invertebrates, including soil insects and earthworms, microorganisms, etc.)

(air, water, soil) and the transport behavior within each medium. A knowledge of physical-chemical parameters such as

- vapour pressure/volatility from aqueous solution;

- water solubility;

- adsorption/desorption;

- complex formation ability;

- density of liquids and solids;

- particle size distribution;

- viscosity of liquids;

- surface tension of aqueous solutions.

allows an estimate of the distribution of a substance between air, water and soil, after its release into the environment.

Volatilization can be the principal means of dissipation in the environment for some chemicals.

The capacity of a chemical to volatilize is related to its vapour pressure and the rate of movement away from the evaporation surface. The layer immediately above the evaporating surface is considered virtually immobile and turbulence of the layer acts to decrease its width and therefore increases the rate of volatilization. Temperature influences volatilization through its effect on the vapor pressure, and evaporation will increase between three and four times for every $10^{\circ} \mathrm{C}$ increase.

The measured vapor pressures (and vapor densities) of a number of commonly known pesticides reveal a wide variety of ranges which extend over about five orders of magnitude from $2000 \mathrm{mPa}$ for EPTC to $0.0085 \mathrm{mPa}$ for atrazine, and for organochlorine compounds from ca. $3 \times 10^{-5} \mathrm{mPa}(\mathrm{PCB})$ to ca. $49 \mathrm{mPa}$ (toxaphene) [8]. However, the vapor pressure of halogenated aliphatic hydrocarbons are much larger, up to $1 \times 10^{4} \mathrm{mPa}$. It appears that no clearcut relationship exists between the values and the chemical structures.

The general scale is however very low in comparison with more familiar volatile organic chemicals such as alcohols or ethers which have vapor pressures close to one atmosphere at normal temperatures. The pressure of standard atmosphere $(760 \mathrm{mmHg})$ is equal to $1 \times 10^{5} \mathrm{~Pa}(1 \mathrm{mmHg}=130 \mathrm{~Pa})$, or about five orders of magnitude greater than the vapor pressure of EPTC. While the values shown above may seem to suggest that the volatilization rates of the compounds would be very low, it must be remembered that their background vapor pressures in the air are essentially zero so that the residues will evaporate as if they were exposed to a vacuum.

The water solubility of chemicals is known to be important in relation to volatility. Thus, the distribution between air and water is described by Henry's law, defined by the equation

$K_{\mathrm{AW}}=C_{\mathrm{A}} / C_{\mathrm{w}}$ 
Where $C_{\mathrm{A}}$ is the vapor density and $C_{\mathrm{W}}$ is the solution concentration. Solubility and Henry's law coefficient of representative pesticides and organochlorine compounds are quite variable, with solubility of $10^{-1}-10^{-3} \mathrm{~g} / \mathrm{m}^{3}$ and $K_{\mathrm{AW}}$ of $10^{-2}-10^{-5}$ for certain organochlorine compounds.

Under the circumstances soil is present, it generally affects the volatilization process. Temperature influences volatilization through its effect on the vapor pressure and additionally it can influence rates of diffusion within soil. Increased soil moisture content acts to increase volatilization by reducing the number of surface active adsorption sites in soil. When dry soil is rewetted, the rates of volatilization will increase. Soils with high organic matter contents are subject to lower volatility simply due to the extent of adsorption of chemicals onto what is ostensibly a solid organic phase. Also chemicals with higher aqueous solubilities are more susceptible to volatilization because of the large role soil-water plays in transport and desorption within soil.

Although the speed at which residues can enter the atmosphere through evaporation is to some degree offset by rapid dilution, re-deposition as a result of adsorption and re-concentration of the vapor in rain or fog droplets is a known pathway that may represent the significant step in the long distance transport of those chemicals and their degradation products that are sufficiently stable in air or water.

Adsorption of neutral molecules by soil is most effectively correlated with organic matter or organic carbon content of the soil. Adsorption per unit of organic carbon $\left(K_{\mathrm{OC}}\right)$, is relatively constant within a two to three fold range for a particular compound in surface soils. A variety of relationships has been developed between $K_{\mathrm{oc}}$ and various physicochemical parameters, the most useful being with the octanol-water partition coefficient $\left(P_{\text {ow }}\right)$.

Thus, as is well-known, $K_{\text {oc }}$ is correlated linearly with $P_{\text {ow }}$ of the chemicals, as exemplified below [9]. $\log K_{\mathrm{oC}}=a \log P_{\mathrm{ow}}+b$

$\begin{array}{llll}\text { compounds } & a & b & (r) \\ 45 \text { pesticides }\left(\log P_{\text {ow }} 2-6\right) & 0.54 & 1.38 & (0.86) \\ \text { 20 PAHs }\left(\log P_{\text {ow }} 1-6\right) & 0.83 & 0.29 & (0.95)\end{array}$

where $\mathrm{PAH}=$ polyaromatic hydrocarbon.

Chemicals applied to soil may move downward through soil profile, and the leaching depends not only upon adsorption and desorption processes, but also upon the structure of the soil, as well as physicochemical properties of soil; the lower the organic matter and mineral matter content and the lower $K_{\text {ow }}$ of chemicals, then the higher sub-surface transport through soil (leaching) is apparent. (NB $\log K_{\mathrm{ow}}$ of selected organochlorine compounds are aldrin 5.14-7.4, chlordane 6.00, DDT 4.89-6.91, dieldrin 3.69-6.2, dioxins 4.75-8.0 furans 5.44-8.0, heptachlor 4.40-5.5, hexachlorbenzene 3.03-6.42 [8]. Therefore, they tend to stay at the surface of soil. In contrast, the $K_{\mathrm{oc}}$ values of several halogenated aliphatic hydrocarbons are by far smaller, suggesting easier leaching potentials.) According to Cohen et al. (1984) a set of certain parameters for leaching (mobility) potential in hydrologically sensitive areas are as follows [10];

1 Water solubility-greater than 30 p.p.m.,

$2 K$ d (soil water distribution constant) - less than 5 and usually less than $1-2$,

$3 K_{\text {oc }}$-less than $300-500$,

4 Henry's law constant-less than $10^{-2} \mathrm{~atm} \cdot \mathrm{m}^{3} / \mathrm{mol}$.

Surface transport or run-off of chemicals occurs in situation where there is a combination of sloping land, a near-by water course and appropriate rainfall. Less organic matter and mineral matter contenct of soil as well as lower solubility and lower $K_{\mathrm{oc}}$ of chemicals in particular increase run-off.

To understand behavior of chemicals in soil, simple techniques such as lysimeter experiment which, by using a certain dimension of core soil, mimic actual environment, have been widely used. More recently several sophisticated mathematical models have been proposed to predict behavior of chemicals in soil, including PELMO (SESOIL) for leaching and PRZM2, EXAMSII, GLEAMS, etc. for runoff [11]. (For concise evaluation of mathematical models for leaching and runoff, see Ref. 11)

\subsection{Bioconcentration and bioaccumulation [12]}

Bioconcentration and bioaccumulation is also closely related with their physicochemical properties. The high concentration of organochlorine insecticides such as DDT and dieldrin in the tissues of avian and mammalian species, and even in humans, results from uptake of these chemicals mainly through food. In 
the case of aquatic fish the compounds will be taken up through gills or epithelial tissues as well. Thus, bioconcentration is defined to be the process by which a compound enters aquatic organisms directly from water through gills or epithelial tissues. Bioaccumulation includes not only bioconcentration but also any uptake processes of chemical residues through food. Biomagnification refers to total process by which tissue concentrations of a bioaccumulated chemical increase as this material passes up through two or more trophic levels.

Adsorption on the outer body surface with subsequent absorption by diffusion is another important mechanism for uptake in micro-organisms such as zooplankton, phytoplankton and bacteria having high surface/volume ratios.

After uptake of a chemical from water through semi-permeable membranes the compound distributes with the circulatory fluid, subject to excretion through gill as well as transformation and storage. Thus, it is easily understood that significant biomagnification occurs for compounds having long half-lives in aquatic organisms, generally corresponding to large bioaccumulation ratios.

The relationships of bioconcentration to partition coefficient has been concluded as follows. If the octanol/water system is a perfect surrogate for the organisms/water system, the slope of the plots (constant $a$ ) in the following equation should be unity [9].

$\log B_{\mathrm{R}}=a \log P_{\mathrm{ow}}+b$

where

$B=$ bioconcentration ratio,

$P_{\text {ow }}^{\mathrm{R}}=$ partition coefficient between octanol and water,

$a=1.00$ for fish, 0.884 for molluscs, 0.855 for mussels, 0.898 for daphnids and 0.907 for microorganisms,

$b=$ reflection of the lipid content of the organism.

The substances with $\log P_{\text {ow }}$ higher than 3 are actually liable to bioaccumulate. And although highly chlorinated compounds tend to bioaccumulate significantly, the presence of chlorine in the molecule is in itself not essential for bioaccumulation. For example, halogenated aliphatic hydrocarbons with $P_{\text {ow }}<3$ are least bioaccumulative. Biotransformation is an important process for the elimination of foreign chemicals. Therefore, for those compounds with measurable biodegradation potential (e.g. the pyrethroid insecticides with $P_{\text {ow }}$ similar to organochlorine insecticides), bioconcentration ratios are significantly lower than those expected from the above relationships obtained mostly from data on chlorinated hydrocarbons.

Since uptake of xenobiotic chemicals occurs by passive diffusion, it is not influenced by the presence of other compounds; this is exemplified by combinations of dieldrin/aroclor 1232, $p, p^{\prime}$-DDT/heptachlor and fenitrothion/methyl parathion.

The biological half-lives of chlorine containing pesticides in fish (mainly trout, catfish, sunfish, minnow and goldfish) are variable; chlordane $\left(t_{1 / 2}, 31-119\right.$ days), $p, p^{\prime}$-DDT (31->409 days), DDE (336 days), dieldrin ( $6-90$ days), heptachlor ( 32 days), mirex (130- $>495$ days), toxaphene (7-63 days); endrin (3-28 days), methoxychlor ( $<2-9$ days); whereas others not so much, endosulfan, lindane, chlorpyrifos, atrazine, benthiocarb, 2,4-D, diflubenzuron, propanil, and permethrin $(<1$ week).

\subsection{Degradation and metabolism $[5,12]$}

Chemicals in the environment may be degraded (transformed) in various ways through abiotic (non-biological) as well as biotic (biological) actions. Hydrolysis and photolysis are two major factors responsible for abiotic degradation.

Chemicals possessing ester and other hydrolyzable linkages (carbamate, amide, nitrile, etc.) in the molecule may be decomposed in aqueous media. The reaction undergoes either through acid $\left(\mathrm{H}^{+}\right)$catalyzed hydrolysis or base $\left(\mathrm{OH}^{-}\right)$catalyzed process, and may yield different hydrolysis products under different pH's.

However, since the $\mathrm{pH}$ range encountered in natural water is between 5 and 9 , hydrolysis is generally of minor importance in the degradation of chemicals in the environment. However, hydrolysis will be important for decomposition of chemicals in ground water where photodegradation proceeds at a negligible rate.

Photolysis is another important factor for abiotic degradation of chemicals in the environment. Photolysis can be divided into direct photolysis and indirect photolysis. Direct photolysis proceeds via direct absorption of sunlight energy followed by chemical reactions. Wavelengths at $290-330 \mathrm{~nm}$ (UV-B) are 
more effective than those longer than $330 \mathrm{~nm}$ (UV-A). (Wavelengths below $290 \mathrm{~nm}$ are absent from the troposphere due to absorption in ozone layer.) The kinetics of direct photolysis are composed of two factors, light absorption and an efficiency term, the quantum yield. Thus, photolysis is dependent on latitude and altitude on the earth as well as seasons.

Indirect photolysis requires a sensitizer and can be generalized by two mechanisms. One of these is the energy transfer process, that is, the sensitizer absorbs light and transfers the energy to another chemical which then undergoes photochemical reactions. Naturally occurring substances such as humic acid and fulvic acid are known as possible sensitizers by the energy transfer mechanism. In the alternative mechanism the light absorption results in homolytic cleavage of a chemical bond with formation of radicals and subsequent reaction of the radicals. Indirect photolysis is not understood well. However, obviously it is important in the transformation of chemicals as described below.

It has been shown that singlet molecular oxygen, ozone, hydroxy radical, peroxy radical, hydrated electron, superoxide radical anion, and hydrogen peroxide are photochemically generated as the reactive chemical species in aquatic environments. Dissolved substances in natural water such as humic acid, phenylalanine, tyrosine and tryptophan are involved in the generation of these reactive species.

Photolysis yields a wide variety of products depending on the conditions mentioned above, as well as the chemical structure of the compounds in question. Photolytic products generally agree to those of chemical and biological transformation. However, a variety of unique reactions is also observed; photoisomerization of pyrethroid insecticides as well as cyclodiene compounds, and decarboxylation of cyano pyrethroids. Also de(hydro)chlorination of DDT proceeds under photoirradiation. The dechlorination of dichlorobenzene, chloroaniline and chlorophenol to monochlorobenzene, aniline and phenol, respectively, is observed, as well as photoinduced dechlorination of PCBs and dioxins. As compared with non-chlorinated compounds, some organochlorine compounds are rather resistant against photolysis, whereas others are decomposed as easily as nonchlorinated compounds.

\section{Dechlorination}

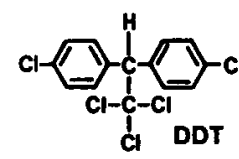

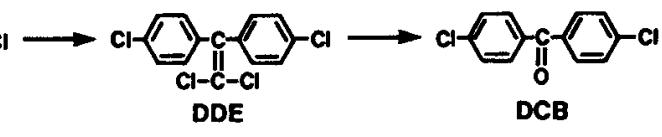

DCB

It is now well known that the major (and sometimes only) mechanism of degradation for many chemicals in the natural environment is by microbial activity. The fact that some compounds used repeatedly in agriculture become less effective as pest control agents is simply because of their quick degradation by soil microflora.

Microbial metabolism is dependent on a variety of factors such as moisture content, organic matter content, $\mathrm{pH}$, temperature, etc. There is usually greater biological activity in moist soils than in dry soils and thus more capacity for biodegradation to take place.

The organic matter in soils has also been found to play an important role in the adsorption and desorption process, and to increase the rate of biodegradation and often corresponds to high native bacterial and fungal populations. This is particularly true with chlorinated pesticides such as DDT, Endrin, and Heptachlor.

The effect of soil $\mathrm{pH}$ on biodegradation varies widely between chemical families. Many $\mathrm{pH}$-related effects on degradation correspond to those variables affecting the activity of certain microorganisms or to the conditions under which certain enzymes are active.

Also redox potential occasionally affects degradation of chemicals in soil, to yield the reduced products. Temperature naturally influences biodegradation because of its effect on microbial and enzyme activities. This phenomenon is well illustrated in the tropics where biodegradation proceeds faster than in temperate soils.

The type of chemical transformation by soil microorganisms are quite variable and widespread; several types of oxidation, hydrolysis, decarboxylation, and isomerization. In particular noteworthy are reductive dechlorination (as well as dehydrochlorination) and fission of aromatic ring.

Under specific conditions by consorted actions of anaerobic (dechlorination) and aerobic (ring oxidation and fission) microbes, even PCBs can be degraded to simple organic compounds, although the rate is not so rapid as to warrant efficient mineralization of all these compounds in measurable terms.

Aquatic organisms including invertebrates and phytoplanktons as well as vertebrates, can carry out a variety of biotransformation reactions, even though they have generally lower overall activities than comparable mammalian systems; a variety of oxidation, hydrolysis, reduction, directly onto the xenobiotics 
Reduction
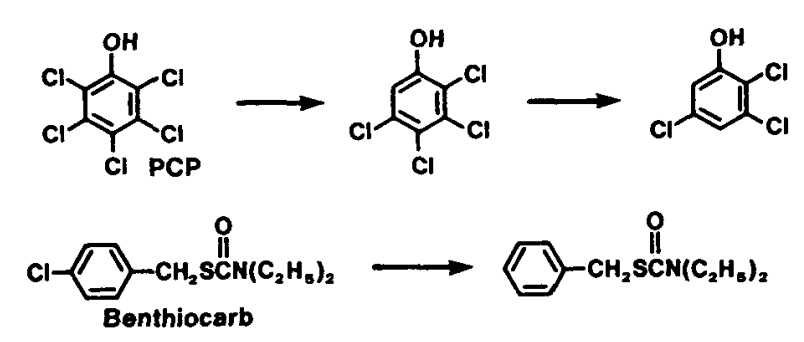

Aromatic ring fission

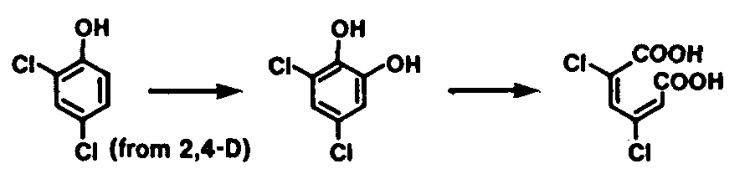

which are commonly termed phase I reactions as well as a wide range of conjugation reactions or phase II reactions (by which primary metabolites are bound with glucuronic acid, sulfate, amino acid, etc.) are documented in these species. A significant difference in biotransformation is observed between taxonomic phyla.

In addition, avian species are quite well known to possess enzymes more less similar to mammals to degrade a variety of chemicals, although quantitatively these two groups of animals are not necessarily comparable.

\subsection{Overall environmental behavior of chemicals [5]}

Once a chemical is released to the natural environment, it is subject to several translocative as well as transformative processes which will eventually remove the parent compound and metabolites as briefly discussed above. However, when chemicals are not transformed rapidly but instead are retained in the environment, these can pose threats to a wide range of organisms not solely due to the toxicity of the agents but often as a result of their bioaccumulation. The most dramatic examples are the persistent organochlorine pesticides. For example, typically DDT at concentrations of 0.000004 p.p.m. in air and 0.00001 p.p.m. in fresh water system results in bioaccumulation, at 10.1 p.p.m. in fish eating birds. Thus, in short,

1 DDT has been used in many areas in the world in large amounts (several ten thousand tonnes/year), for half a century,

2 is sparingly water-soluble and highly lipophilic, with $P_{\text {ow }}$ of ca. $10^{6}$ and stable against photolysis and microbial degradation,

3 is gradually evaporated into air, which results in a wide range transport, together with extensive contamination of river and sea, and,

4 is easily absorbed and translocative to adipose tissues of living organisms.

$5 \mathrm{Cl}-\mathrm{C}$ (aromatic) bond of DDT is resistant against metabolic attack, and

6 no other linkages in the molecule are subject to easy metabolic attack. This might be equally characteristic of DDT.

Moreover,

7 DDT and its metabolites (e.g. DDE) develop a certain range of chronic and/or reproductive toxicities in living organisms, such as disturbance of Ca metabolism in avian species resulting in thinning of egg shell, and induction of neoplasms in rodent tissues.

Thus, DDT is quite unique in these characteristics. Exceptionally few limited number of chemicals possess physicochemical properties and biological activities more or less comparable to DDT, and many organochlorine compounds are either readily or inherently biodegradable, resulting in different environmental behaviors from those of DDT.

The degree of persistency of organic chemicals (and their potential to bioaccumulate) is considered already by The Pesticide Chemistry Commission, IUPAC [13]. The Commission defines that persistence is the residence time of a chemical species in a specifically defined compartment of the environment, as affected by physical and chemical properties of the compound, composition and characteristics of relevant environmental compartment, as well as climatic conditions. Thus, measurement of the overall degradation rate of a chemical and all the various processes affecting its disappearance from the relevant compartment defines persistence in either specific or comparative terms. 


\section{ECOTOXICOLOGY AND ENVIRONMENTAL RISK ASSESSMENT [14,15]}

\subsection{Ecotoxicity testings}

Damage to the biotic part of an ecosystem may impair the structure and function, and affect the integrity of the ecosystem. In the functioning of ecosystems, trophic relations, involving energy and nutrient transfers, which are part of natural cycles, are of prime importance.

In this respect, the functional distinction between primary producers, secondary producers, consumers and decay organisms is useful in visualizing important biotic elements of these natural cycles. Environmental disturbances will generally manifest themselves as changes in relative numbers or as changes in the biomass of elements of the system or as both. Biodepressive effects (mortality, decrease of growth and reproduction) and biostimulatory effects (eutrophication, immigration, etc.), which may both be harmful to the ecosystem and as such undesirable, may be distinguished.

Table 2 is a rough summary of the relative importance of the environmental compartments to the ecosystem, implying that their relative importance depends on whether the environmental compartments are looked at as a habitat, as a transport medium or as a sink for chemicals [14].

One of the main difficulties in ecotoxicology is to decide whether or not some observed change is ecologically significant, and this may involve difficulties for extrapolation.

Ecologically irreversible changes are considered to be undesirable effects of pollution; reversible effects may, however, also be objectionable, although they may be tolerated under certain circumstances. The ecological importance of an effect may differ widely between species, e.g. because of differences in reproduction rate or life cycle span.

For the assessment of environmental effects of chemicals, ideally the whole ecosystem should be considered; for practical and scientific reasons, however, the analysis of the effects on subsystems may only be feasible.

Also difficulty lies in how to predict the impact of very low concentrations of a chemical, maintained for long periods, and affecting communities of organisms, giving rise to sublethal and interspecific phenomena. Ideally chronic tests with multi-species systems should be carried out, but no standard test systems of such a nature are available, nor they are feasible, because of an enormous task. Therefore, it is generally agreed that a stepwise procedure (tier system) is to be followed, starting with simple tests for all compounds but terminating with more complex multispecies and field tests for those few compounds which are of great practical value, and which have been indicated as being potentially dangerous to the environment.

Among a variety of species as well as test procedures being utilized in many countries for screening purposes, several testings are suggested by the OECD Guidelines on Ecotoxicology [14].

These testings to be mentioned below have been selected based on the analyses of a fairly large amount of experiments carried out in the past, and generally the results of testings may be considered to be usable as surrogates giving rough approximations in other environmental situations. Thus, it should be stressed that all tests serve only as models and consequently have shortcomings. Prediction of all possible environmental risks cannot be expected directly from these tests.

Table 2 The relative importance of the environmental compartments air, water and soil in relation to some of their properties

\begin{tabular}{|c|c|c|c|c|c|c|c|}
\hline & $\begin{array}{l}\text { Content } \\
\text { of biotic } \\
\text { systems } \\
\text { (habitat) }\end{array}$ & $\begin{array}{l}\text { Transport me } \\
\text { pollution } \\
\text { Distance of } \\
\text { transport }\end{array}$ & $\begin{array}{l}\text { edium for } \\
\text { Volume } \\
\text { transported }\end{array}$ & $\begin{array}{l}\text { Risk from } \\
\text { chemicals } \\
\text { to populations } \\
\text { present }\end{array}$ & $\begin{array}{l}\text { Sink for } \\
\text { pollution }\end{array}$ & $\begin{array}{l}\text { Global } \\
\text { uniformity } \\
\text { of } \\
\text { composition }\end{array}$ & Dilution \\
\hline Air & + & +++ & ++ & + & + & +++ & +++ \\
\hline Water & + & ++ & +++ & +++ & +++ & $+t$ & $+t$ \\
\hline Soil & +++ & + & + & ++ & +++ & + & + \\
\hline
\end{tabular}

+ slight,++ moderate, +++ considerable. 


\subsection{Environmental risk assessment}

The primary objective of ecotoxicology studies is to execute environmental risk assessment. (For general discussion on risk assessment, refer to Section 4.) Two parameters are needed as essential for the assessment; one is PEC (predicted environmental concentration of the chemical to be assessed in the eco-compartment in question.) The other parameter is PNEC (predicted no effect concentration of the chemical to biota in the eco-compartment). If risk factor defined as PEC/PNEC is less than $1(\mathrm{PEC} / \mathrm{PNEC}<1)$, then the risk of the chemical is insignificant. Under circumstances $\mathrm{PEC} / \mathrm{PNEC}>1$, there may be undue risk to the ecosystem, and steps for risk reduction should be forthcoming.

Although actual environmental monitoring may be occasionally carried out to get PEC, in most cases such is not feasible, and PEC will be assessed (by calculation) based mainly on certain assumptions regarding production volume, usage and application, emission, eco-compartments to be considered (air, water, soil, etc), as well as physicochemical properties and information on environmental behavior of the chemical.

It is even more difficult to get PNEC, because ideally PNEC should cover all the living organisms in the eco-compartment in question, although the evaluation of PNEC is made often based on the toxicity information on a limited number of organisms not found in there. In the absence of experimental data, the toxicity figure may be determined by QSAR (quantitative structure activity relationship) extrapolation of group of structurally similar compounds. Anyhow, since certain extrapolations are needed to bridge the gaps mentioned above, the application factor (uncertainly factor) has been elaborated. For example, U.S. EPA and EU guidelines suggest the following application factors to calculate PNEC;

- acute toxicity figure of one species, $\times 1 / 1000$;

- acute toxicity figure of three species, $\times 1 / 100$ (of most susceptible species);

- from acute toxicity to subacute toxicity, $\times 1 / 10$.

etc.

It is quite understandable that the magnitude of application factors should be modified upon accumulation of more toxicity information as well as evaluation of its significance.

The environmental risk assessment should be conducted stepwisely, taking into account all the relevant database, as briefly described below.

\section{Substance characterization}

1 production and importation volume

2 use, application, disposal

3 physicochemical properties, environmental stability/-reactivity

\section{Fate evaluation/environmental release}

1 actual monitoring

2 calculation by models (e.g. McKay model)

\section{Primary ecotoxicity testing}

1 QSAR calculation

2 biodegradation, bioaccumulation

3 acute testing in fish, daphnia, algae

4 acute and subacute testing in aquatic organisms, earthworm, birds, mammals
4. Risk evaluation
1 derivatization of PEC
2 derivatization of PNEC
3 calculation of risk factor (PEC/PNEC)

\section{Supplementary laboratory tests and environmental monitoring}




\section{MAMMALIAN TOXICOLOGY AND RISK ASSESSMENT IN HUMANS}

\subsection{Outline of current procedures}

Any chemicals, natural or anthropogenic, exert toxicity at and above certain dosage, and the type of toxicity is variable depending on their chemical structure. The OECD expert groups have been devoted to produce a well defined framework for each toxicity test which can be used in a similar manner throughout the world [16]. As results they have published guidelines for acute, subacute toxicity tests through different administration routes, tests on local effects on skin and eye, allergic sensitization, delayed neurotoxicity, teratology, reproductive/developmental toxicity (Guidelines 401-421 (1981-1995)) and chronic toxicity and carcinogenicity tests (Guidelines $451-453$ (1981)). The guidelines for a range of mutagenicity tests with in vitro and in vivo systems have been established as well (Guidelines 471-485 (1983-1986)). In addition, toxicokinetics is included (Guideline 417), which, defined as the study of the rate of absorption, distribution, metabolism and excretion of toxic substances, is useful for selecting appropriate dose levels in chronic studies based on dose-dependent kinetics, and also for evaluating test results from toxicology studies to be extrapolated to man. To ensure quality and integrity of all these laboratory studies for registration purposes, the GLP (Good Laboratory Practice) guidelines are introduced in many countries. Because of a large number of chemicals with no sufficient toxicology information, QSAR approaches are intensively pursued to fill the data gaps, although satisfactory results have been acquired only occasionally to date. These toxicity data, together with other relevant information, constitute hazard identification, first step in the whole risk assessment procedure.

Figure 1 shows the risk assessment procedure proposed by U.S. National Academy of Science in 1983, which is used worldwide today as addressing the standard concept of risk assessment. In this scheme the second step which follows hazard identification, dose response assessment, is to execute two different types of extrapolation, one from high to low dose, and the other from experimental animal species to man. Since both extrapolations together leading to safe dose for man, are generally very difficult to make rationally, one of the procedures to be taken customarily by many national regulatory agencies as well as by WHO is to follow so-called AD1 (acceptable daily intake, or RfD, reference dose) approach, in which NOEL (no observed effect level) or NOAEL (no observed adverse effect level) obtained by long-term studies (e.g. chronic toxicity, carcinogenicity, teratology and reproductive toxicity) in most susceptible

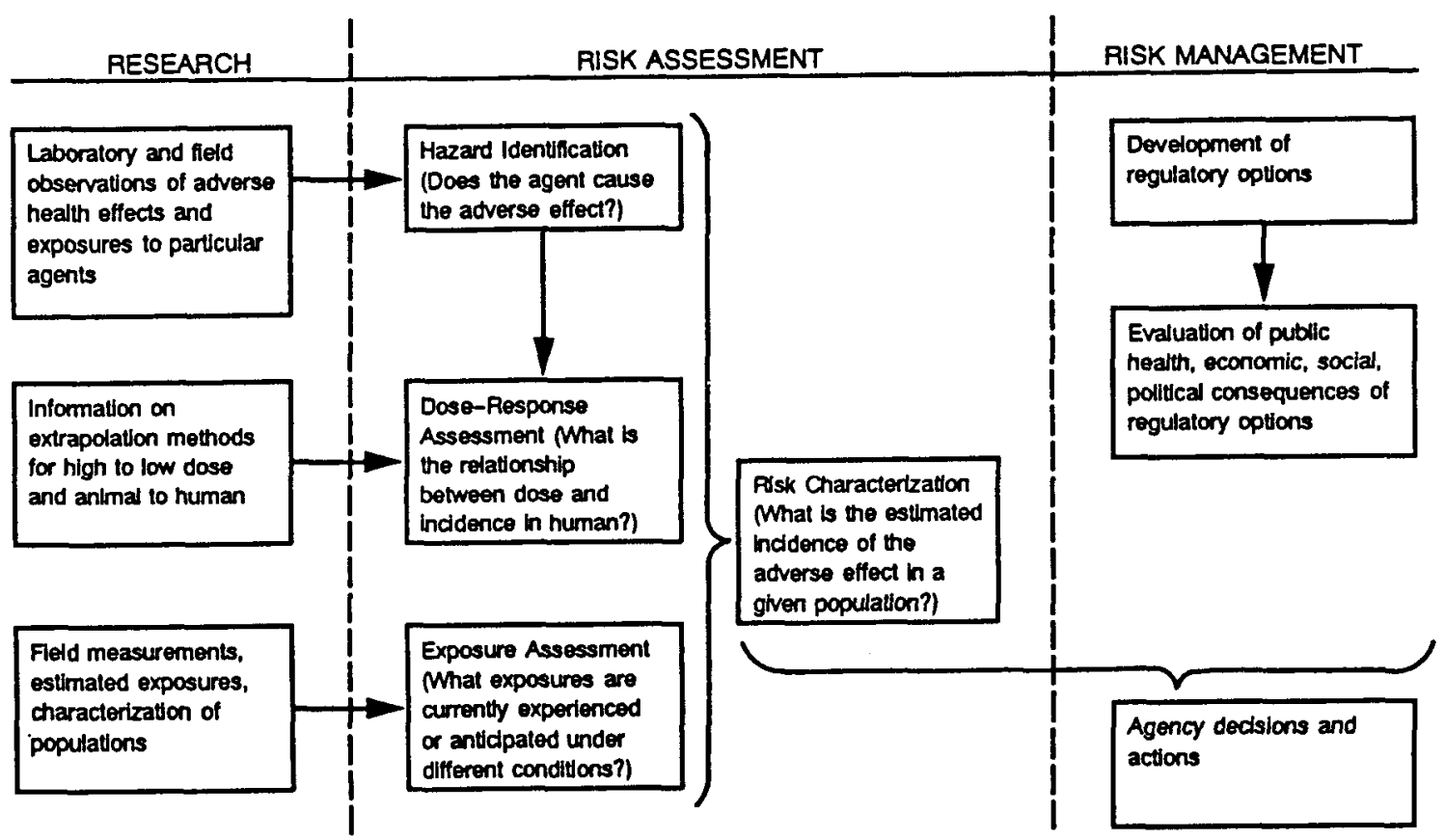

Fig. 1 Elements of risk assessment and risk management (from National Academy of Science, 1983). 
animals is multiplied by safety factor or uncertainly factor: AD1, $\mathrm{mg} / \mathrm{kg} \mathrm{man} / \mathrm{day}=$ NOEL (NOAEL) $\times$ safety factor. Recently, the use of benchmark dose is recommended instead of NOEL (NOAEL), which is defined as a statistical lower confidence limit on the dose producing a predetermined level of change in adverse response compared with the response in untreated animals. For the detailed discussion, see Ref. 17. The magnitude of safety factor will vary depending on the database (whether or not the chemical is extensively studied or when the results suggest serious health effects). Usually the safety factor applied is $1 / 100$, but occasionally, in the order of $1 / 1000$ in e.g. carcinogens, the magnitude which is decided by scientific judgement among experts.

Thus, AD1 is the maximum calculated amount of chemicals to be ingested daily through any exposure routes (through skin, by inhalation or through food and drinking water.) without manifestation of any significant adverse effects. Occasionally, ratio of NOEL/human exposure, or margin of safety, MOS, is calculated. If MOS is large enough, e.g. $>100$, then such a use pattern is justified. In case where it is postulated that the accumulated smallest carcinogenic effects will eventually be made observable, or in other words no threshold exists for carcinogenic potential, then for practical purpose the new concept is to be introduced, where tiny amounts of carcinogenic substance corresponding to the very low rate of the incidence, say $10^{-6}$ or one individual per one million population, shall be allowed. The amounts of carcinogenic substance thus specified is called VSD or virtually safe dose. However, because such tiniest incidence could not be obtained experimentally, some mathematical models shall be elaborated based on extrapolation of the dose-response curve experimentally drawn. Several different models have been proposed to date, assuming either that the sensitivity of individual in the population to carcinogens is presumed to follow a certain distribution (e.g. probit model) or that onset of carcinogenicity follows a certain mechanism (e.g. multi-stage model). However, no existing model has been unanimously accepted by scientists because of several reasons.

Under certain circumstances exposure assessment can be done with the specific population, and actually a variety of human monitoring studies (epidemiology) have been carried out, to establish cause-effect relationship between certain adverse effects in human population and exposure to the specific substances/ factors. These human monitorings are invaluable to be complementary to animal studies, and/or to clarify the specific reaction in man. Now, by combining the above information on hazard identification, doseresponse assessment, as well as exposure assessment, risk characterization can be made, in which incidence of possible adverse effects in a given population can be estimated. Therefore, as is easily understood, risk characterization is inherently probability judgement, quite apart from the mere yes or no (dichotomous) judgement that reflects indeed the reality of life. On the other hand, in risk management economic, social and political consequences have to be duly considered, and by integrating these factors with scientific judgement on the risk (risk assessment) regulatory options should be developed. Therefore, inevitably regulatory option may not be the same between two societies, regardless of the identical scientific risk information. A better social (public) acceptance of risk through accurate risk communication is thus essential for sound regulatory decision.

One of the pitfalls in risk communication is the biased understanding that synthetic chemicals only are hazardous, and natural products are absolutely safe. As is well known, the reality is there are so many natural constituents that are hazardous to human health. Tomatin (tomato), solanin (potato), patulin (apple), tetrodotoxin (swellfish), okadaic acid (shellfish), aflatoxins and ochratoxins (both mycotoxins) are just a couple of such examples. To be worse, sometimes the amounts surpass the safe level to man. In this regard, U.S. National Research Council concludes that analysis of more than 200 chemicals including 65 naturally occurring substances reveals no clear difference in their range of potency in causing cancer in animals. Furthermore, given the greater abundance of natural substances in the diet, the report states, humans are exposed to many more naturally occurring chemicals that might be carcinogens than they are to synthetic carcinogens [18].

\subsection{More rational approach}

Owing to the remarkable progress in scientific disciplines concerned with environmental safety and health, the above process of risk assessment has tended to be modified in several respects.

Needless to say, when chemicals foreign to the normal biochemistry of the body (usually called xenobiotics) are introduced, they undergo metabolic conversion by a battery of enzymes. These metabolic 
reactions majorly lead to detoxication, whereas some products are more biologically active than the parent compound (active metabolite), and toxic response ensues when it reacts with the essential cellular site (DNA, enzyme, receptor, etc.) [19].

Thus, the species difference (and individual difference) in toxicity manifestation results from the metabolic difference as well as the dissimilar response in cellular sites.

A majority of the instances of bioactivation are catalyzed by a family of monooxygenase enzymes often termed mixed function oxidases (or monooxygenases), the hemoprotein cytochrome P450 (CYP), polysubstrate monooxygenases. The P450s are widely distributed in vertebrates, insects, plants and microbes, and located primarily in the endoplasmic reticulum of many tissues, liver, lung, pancreas, intestine, etc. in mammals, with the liver of particular significance quantitatively. There are many isoforms of CYP450dependent monoxygenases (superfamily), with a total of about 220 and more than 80 even present in mammals [20]. The available information indicates that CYP1 A1, 1 A2, 2C $(8,9,18,19), 2 \mathrm{E} 1$ and $3 \mathrm{~A} 4$ are main isozymes in human liver, whereas in rat liver CYP3A2, 2C6, 2C11, 2C13 and 2E1 are predominant [21]. (NB Also see Ref. 22 for role of human P450s in metabolic activation of carcinogens and toxins.) As is widely recognized, each CYP enzyme in different species may have different substrate specificity [20]. Since P450 mediated oxidation of PAHs (polyaromatic hydrocarbons such as benz(a)pyrene and anthracene), heterocyclic amines, butadiene, vinyl chloride and aflatoxins, etc. is prerequisite for their carcinogenicity, such a differential metabolism will have a significant bearing on the carcinogenic potency. And there are growing evidences accumulated to suggest this presumption. Although not mentioned here, such variabilities among species are also seen in other enzymes. Now that human in vitro metabolism information can be incorporated into the so-called PB-PK model (physiologically based pharmacokinetic model) to mimic human metabolism in vivo, by comparison with animal metabolism data more rationalized criteria may be found to select better species for toxicity testing.

A lot of evidences are being accumulated in recent years indicating that inherent difference among species and strains other than differential metabolism are also responsible for biological activity of hormones and toxic effects of xenobiotic chemicals, and molecular approaches for elucidation of the species difference among animals including man are in tremendous progress.

Among these inherent differences other than metabolism is also included the specificity in a number of receptors. The various biological and toxicological responses such as teratogenesis, immune disorder, tumor promotion and induction of drug metabolizing enzyme caused by groups of chemicals including heterocyclic amines, polycyclic aromatic hydrocarbons and halogenated aromatic hydrocarbons are considered to be mediated by a receptor protein designated arylhydrocarbon receptor (AhR). The genetic difference in the susceptibility of mouse strains to these chemicals is governed by polymorphism of AhR, and AhR from responder (C57BL6) with higher susceptibility to TCDD tumorigenesis and non-responder (DBA/2 J) mice reveals structural as well as functional differences; the higher binding affinity (6 times) of C57BL6 AhR to TCDD is related with modification of the specific amino acid sequences in AhR protein structure.

The human AhR has been known to resemble DBA/2 J AhR in terms of structure and TCDD-binding activity [23].

In addition to diversity of estrogen receptor and androgen receptor related to well known differential activities of the various agonists and antagonists, such polymorphism of AhR is definitely closely associated with similarity and difference among species in the response to a range of toxic chemicals including TCDD.

One of the most important things to recognize during risk characterization is that however precisely the sophisticated in vitro systems work to lead apparently to persuasive conclusions, they inevitably lack dynamic, homeostatic mechanism that is functioning in ordinary in vivo status. Therefore, such in vitro results should be handled with utmost precaution when extrapolation to in vivo situation is attempted.

The monitoring of biomarkers (functional, physiological, biochemical, or molecular) in human population is used to clarify interaction between a potentially hazardous chemical and a biological system [24].

Usually, blood or urine sample (and occasionally breath) is used for biological monitoring, to measure metal (ion), parent compound and metabolites - benzene, toluene, PCB, DDT, acetone (from 2-propanol), mandelic acid (from styrene), and phenol (from benzene). The (inhibition of) cholinesterase (by organophosphorus insecticides) is one example of functional biomarkers. Thus, a number of biomarkers are used for nephrotoxicity, liver toxicity, immunotoxicity and pulmonary toxicity. 
The importance of biomarkers in assessing carcinogenesis of chemicals is also being raised. In addition to cytogenetic changes including chromosomal aberration and sister chromatid exchanges, DNA adducts and protein (hemoglobin) adducts are measured in lymphocytes as indicaters of exposure to carcinogens [25]. Also neurophysiological and neurobehavioral parameters as well as biomarkers of reproductive and developmental toxicity should be included as particularly important as clues to clarifying certain adverse effects of chemicals on man.

These biomarkers can be effectively used for assessing more precisely hazard identification, doseresponse relationship, as well as degree of exposure in humans. And as such the results are quite useful for human epidemiological studies.

Overall, these cellular and molecular approaches including certain aspects of human studies mentioned above, therefore on comparative aspects, contribute significantly to solve the species difference between animals and man, and to establish more scientifically justifiable extrapolation from experimental animals to man, by replacing somewhat controversial existing strategies. In addition, the use of appropriate biomarkers applied to man will enable exposure analysis more precise. Thus, the modern multidisciplinary toxicology sciences will furnish sound basis for more reliable risk characterization.

\section{REFERENCES}

1 Eurochlor; Halogenated Organic Compounds in the Environment (1994).

2 OECD. Guidelines for Testing of Chemicals. Section 1. Physical chemical properties, GL 101-117 (1981, 1995).

3 OECD. Guidelines for Testing Chemicals. Section 3. Degradation and accumulation, GL 301-306 (1981, 1992).

4 A.W. Taylor. in Progress in Pesticide Biochemistry and Toxicology, edited by T.R. Roberts, P.C. Kearney Vol. 9, pp. 257 (1995).

5 N. Mackay, W.B. Betts. in Biodegradation, edited by W.B. Betts, pp. 1. (1992).

6 P.R. Fisk. in Progress in Pesticide Biochemistry and Toxicology, edited by T.R. Roberts, P.C. Kearney Vol. 9. pp. 1. (1995).

7 D.J. Arnold, G.G. Briggs. in Progress in Pesticide Biochemistry and Toxicology, edited by D.H. Hutson, T.R. Roberts. Vol. 7, pp. 101 (1990).

8 IPCS. POPS Assessment Report (1SG/96.5B)(Dec. 1995).

9 OECD. Environmental Monographs. No. 67 (1993).

10 S.Z. Cohen. in Progress in Pesticide Biochemistry and Toxicology, edited by D.H. Hutson, T.R. Roberts. Vol. 7 , pp. 13 (1990).

11 S.Z. Cohen, R.D. Wauchope, A.W. Klein, C.V. Eadsforth, R.D. Jones. Pure and Applied Chemistry 67, 2109 (1995).

12 J. Miyamoto, N. Mikami, Y. Takimoto. in Progress in Pesticide Biochemistry and Toxicology, edited by D.H. Hutson, T.R. Roberts Vol. 7, pp. 123 (1990).

13 R. Greenhalgh. Pure and Applied Chemistry 52, 2563 (1980).

14 OECD. Guidelines for Testing Chemicals Section 2. Effects on Biotic systems, GL, 201-210 (1984, 1992).

15 OECD. Guidelines for Testing Chemicals Section 2. Effects on Biotic Systems. Summary of Considerations in the Report from the OECD Expert Group on Ecotoxicology (1993).

16 OECD. Guidelines for Testing Chemicals-Section 4. Health Effects, GL 401-421, 451-453, 471-485, (19811995).

17 U.S. EPA. Risk Assessment Forum (EPA/630/R-94/007) (Feb. 1995).

18 U.S. National Research Council. Carcinogens and Anticarcinogens in Human Diet; A Comparison of Naturally Occurring and Synthetic Substances (1996).

19 B.M. Hollingworth, N. Kurihara, J. Miyamoto, S. Otto, G.D. Paulson. Pure and Applied Chemistry 67, 1487 (1995).

20 J. Jacob. Pure and Applied Chemistry 68, 301 (1996).

21 T. Shimada, H. Yamazaki, M. Mimura, F.P. Guengerich. Abstract of 23rd Symposium on Xenobiotic Metabolism and Efficacy/Toxicity of Drugs (Kyoto), 29-S-5 (1992).

22 F.J. Gonzalez, H.V. Gelboin. Drug Metabolism Reviews 26, 165 (1994).

23 M. Ema, N. Ohe, M. Suzuki, J. Mimura, K. Sogawa, S. Ikawa, Y. Fujii-Kuriyama. J. Biol. Chem. 269, 27337 (1994).

24 IPCS. Environmental Health Criteria. Vol. 155 (1993).

25 IARC. Monograph on the Evaluation of Carcinogenic Risks to Humans. Vol. 60 (1994). 